\title{
Protective effect of nebivolol on doxorubicin-induced cardiotoxicity in rats
}

\author{
Enas Ahmed Mohamed ${ }^{1}$, Hussien H. Kassem²
}

${ }^{1}$ Department of Anatomy and Embryology, Faculty of Medicine, Cairo University, Cairo, Egypt

2Department of Cardiology, Faculty of Medicine, Cairo University, Cairo, Egypt

Submitted: 26 September 2016

Accepted: 24 November 2016

Arch Med Sci 2018; 14, 6: 1450-1458

DOI: https://doi.org/10.5114/aoms.2018.79008

Copyright (c) 2018 Termedia \& Banach

\author{
Corresponding author: \\ Enas Ahmed Mohamed \\ Department of Anatomy \\ and Embryology \\ Faculty of Medicine \\ Cairo University \\ Kasr Ai Aini \\ 45321 Cairo, Egypt \\ Phone: 02011111124431, \\ 0201001181061 \\ E-mail: indicid1@yahoo.com
}

\section{Abstract}

Introduction: The cardiotoxicity of doxorubicin is incompletely understood. We investigated the prophylactic effect of nebivolol on doxorubicin-induced cardiac toxicity.

Material and methods: Thirty rats were divided into a control group, doxorubicin-treated group and nebivolol + doxorubicin-treated group. The specimens were examined using $\mathrm{H}+\mathrm{E}$ and Masson's trichrome, caspase 3, endothelial nitric oxide synthase (eNOS), inducible nitric oxide synthase (iNOS) and tumor necrosis factor factor- $\alpha$ (TNF- $\alpha)$. The mean area percentage of collagen fiber content, caspase- 3 , eNOS, iNOS and TNF- $\alpha$ immunoactivities was measured.

Results: The doxorubicin-treated group showed marked myocyte distortion and fragmentation, congestion and cytoplasmic lysis in most fibers. These changes were less intense in the nebivolol-treated group. The mean area percentage of collagen fiber in the nebivolol-treated group was non-significantly smaller $(p=0.07)$ than that in the doxorubicin-treated group. The expression of caspase-3 $(p=0.03)$, eNOS $(p \leq 0.001)$, iNOS $(p<0.001)$ and TNF- $\alpha(p=0.003)$ immunoreactivity was improved in the nebivolol-treated group.

Conclusions: Nebivolol exerted a significant protective effect from doxorubicin toxicity. The protective effect appears to be mediated mainly through caspase- 3 , eNOS, iNOS and TNF- $\alpha$ modulation.

Key words: Wistar male rats, doxorubicin, nebivolol, cardiotoxic, nitric oxide synthase caspase apoptosis.

\section{Introduction}

Anthracyclines are among the most potent chemotherapeutic agents. However, cardiotoxicity remains one of their major limitations. More than $50 \%$ of patients exposed to anthracyclines demonstrate some evidence of cardiac dysfunction up to 20 years after the completion of chemotherapy. Approximately $5 \%$ will develop overt heart failure and this incidence may reach $27 \%$ if trastuzumab was added to the initial chemotherapy regimen [1].

Established doxorubicin cardiomyopathy could be lethal, with mortality rates approaching $50 \%$ if clinical heart failure develops. The problem is compounded by the lack of effective treatment for established doxorubicin cardiomyopathy [2]. 
The exact mechanisms underlying doxorubicin cardiotoxicity are not totally understood. Oxidative stress is partly responsible for the cardiotoxicity as the heart does not possess the necessary antioxidant mechanisms [3]. Other potential mechanisms include induction of apoptosis, abnormal extracellular matrix deposition and mitochondrial iron overload [4]. $\beta$-Adrenergic blockers can provide some protection from this toxicity [1] Particularly third-generation $\beta$-blockers are appealing. Nebivolol has an antioxidant effect and vasodilating properties ascribed to its interface with the L-arginine/nitric oxide pathway [5]. Some researchers have discussed the protective effect of nebivolol on doxorubicin-induced cardiotoxicity both in rats and humans. Imbaby et al. found that administration of nebivolol attenuated both the functional and histopathological picture of doxorubicin-induced cardiotoxicity [6]. Kaya et al. found that nebivolol treatment protects the myocardium against anthracycline-induced cardiotoxicity in breast cancer patients [7].

We sought to examine whether pretreatment with nebivolol would protect from the cardiotoxic effects of anthracyclines in a murine model of doxorubicin cardiotoxicity and to probe the possible mechanisms of such protection. We considered extracellular matrix deposition as seen Masson trichrome stain and endothelial nitric oxide synthase (eNOS) and inducible nitric oxide synthase (iNOS) as markers of reactive oxygen species generation. We also considered caspase-3 and tumor necrosis factor $\alpha$ (TNF- $\alpha$ ) as markers of apoptosis and inflammation.

\section{Material and methods}

\section{Preparation of drugs}

Doxorubicin hydrochloride (Adriablastina $50 \mathrm{mg}$ vials, Farmitalia Italia S.P.A, Italy) was dissolved in physiological saline.

Nebivolol (Berlin-Chemie AG, Germany) was supplied in the form of tablets (5 mg each). Each tablet was dissolved in physiological saline at a final concentration of $20 \mathrm{mg} / \mathrm{ml}$ and kept at $+4^{\circ} \mathrm{C}$ for a maximum of 4 days.

\section{Animals}

Thirty adult male Sprague Dawley rats aged weighing 1500-1800 g were used in this study. They were obtained from the animal house, Faculty of Medicine, Cairo University. The animals were housed in separate cages (five rats/cage) under standard environmental and laboratory conditions (allowed free water supply and fed ad libitum, temperature $20^{\circ} \mathrm{C}$ and 12 : 12 -h light-dark cycle). We monitored animals daily for weight. Their activities were followed up. They were treated in ac- cordance with the international guidelines for the care and use of laboratory animals and the experiment protocol was agreed by the Ethics Committee, Faculty of Medicine, Cairo University.

The rats were divided into two groups: control and experimental groups.

The control group: included 10 rats that were injected intraperitoneally with $1 \mathrm{ml} / \mathrm{kg}$ physiological saline at the corresponding time as the experimental group ( 5 rats for 2 weeks and the other 5 for 4 weeks).

The experimental group included 20 rats. This group was subdivided into two subgroups 10 rats each.

- Subgroup I: The rats were injected intraperitoneally with doxorubicin in six equal injections every other day for 2 weeks only.

- Subgroup II: The rats received intraperitoneal nebivolol daily for 2 weeks followed by 2 weeks of doxorubicin (total 4 weeks) in the same doses and through the same route as used for the other groups.

Doxorubicin hydrochloride $(2.5 \mathrm{mg} / \mathrm{kg}$ body weight) [8] and nebivolol (4 mg/kg body weight) [9] were liquefied in physiological saline. At the end of the experiment, the rats were sacrificed by intraperitoneal injection of ketamine hydrochloric acid $(100 \mathrm{mg} / \mathrm{kg})$. The thoracic cavity was quickly opened, exposing the still beating heart, which was then excised and processed for histological and immunohistochemical studies. All the results of the Image analyzer study were done by blind researchers.

\section{Light microscopic study}

Parts of the myocardium of the left ventricle were kept in $10 \%$ solution formaldehyde solution (as a fixative) for $72 \mathrm{~h}$. Tissues were then embedded in paraffin blocks. Sections of $5 \mu \mathrm{m}$ thicknesses were obtained from the paraffin blocks and subjected to the following techniques:

1) Histological examination: using hematoxylin and eosin $(\mathrm{H}+\mathrm{E})$ and Masson's trichrome stains for studying the collagen fiber distribution [10].

2) Immunohistochemical staining: for caspase-3, eNOS, iNOS and TNF- $\alpha$ antigens using the avidin-biotin peroxidase complex technique [11]. The sections were collected on poly-L-lysine coated slides. Nonspecific endogenous peroxidase activity was blocked by treatment with $0.9 \%$ hydrogen peroxide in absolute methanol for $10 \mathrm{~min}$. Then, antigen retrieval was done by heating the sections in $10 \mathrm{mM}$ sodium citrate buffer, in a water bath at $95-100^{\circ} \mathrm{C}$ for $30 \mathrm{~min}$. Sections were rinsed two times in PBS Tween 20 for $2 \mathrm{~min}$, then blocked with 5\% normal goat serum for $30 \mathrm{~min}$ at room temperature. Sections were incubated with the primary an- 
tibodies for 30 min, caspase-3 rabbit polyclonal antibody IgG (ab 2302, Abcam, Cambridge, UK), eNOS rabbit polyclonal antibody IgG (ab 5589, Abcam, Cambridge, UK), iNOS rabbit polyclonal antibody IgG (ab15323, Abcam, Cambridge, UK) and tumor necrosis factor alpha antibody rabbit polyclonal antibody IgG (ab6671, Abcam, Cambridge, UK).

Section were incubated with a biotinylated goat anti-polyvalent secondary antibody for $60 \mathrm{~min}$ at room temperature. Immunodetection was carried out with the horseradish peroxidase-avidin-biotin complex method using a VECTASTAIN1 Elite ABC kit (Vector Laboratories Inc., Burlingame, $C A$ ) and DAB was applied as the chromogen. Localization was detected with $D A B$ and counter-stained in Meyer's hematoxylin, dehydrated, and mounted. Negative control sections were done with the same procedure stated before except that the primary antibody was replaced with a non-immune mouse serum. The sections were studied and photographed using a Canon digital camera attached to an IBM computer system.

\section{Image analyzer study}

The studied sections were examined using a Leica LAS V3.8 image analyzer computer system (Switzerland). The mean area percentage of the collagen fiber content of the myocardial sections as well as the mean area percentage of immune reaction for caspase-3, eNOS, INOS and TNF- $\alpha$ was measured using the grey measure menu in 10 non-overlapping fields in each specimen using an objective lens of magnification of 40 (a total magnification of 400 after grey calibration) using the color detect menu and in relation to the standard measuring frame of $7099.95 \mu \mathrm{m}$.

\section{Statistical analysis}

The quantitative data were first examined by the Kolmogorov-Smirnov test for normality. Quantitative data are expressed as mean \pm standard deviation. The data were collected and studied using analysis of variance (one-way ANOVA) followed by the post hoc Bonferroni test for pairwise comparisons among the experimental groups. Statistical tests were done using SPSS 17 (Statistical Package for Social Sciences), Windows Version 9, Chicago USA. Significance was considered at a $p$-value $<0.05[12]$.

\section{Results}

\section{Histological results}

\section{Hematoxylin and eosin-stained sections}

The cardiac myocytes in the left ventricles of the control group showed normal histological ar- chitecture with acidophilic sarcoplasm and central oval nuclei (Figures 1 A, B). Blood capillaries were evident in the intercellular spaces (Figure 1 A). Examination of the ventricular sections of rats of subgroup I revealed marked distortion and fragmentation of cardiac muscle fibers (Figures $1 \mathrm{C}, \mathrm{E}$ ). Some myocytes showed peripheral nuclei (Figure $1 \mathrm{C})$ and pyknotic nuclei (Figure $1 \mathrm{C}$ ). Mononuclear cellular infiltration was present between the cardiac myocytes (Figure $1 \mathrm{E}$ ). Cytoplasmic vacuolation was present in most cardiac muscle fibers (Figures $1 \mathrm{D}, \mathrm{F}$ ) as well as massive congestion of the blood vessels (Figure $1 \mathrm{D}$ ). Dilated lymph vessels were noticed (Figure $1 \mathrm{~F}$ ). The histological pattern of group II was close to that of the control group (Figure $1 \mathrm{G}$ ). Some congested capillaries were observed (Figure $1 \mathrm{H}$ ).

\section{Masson's trichrome stain}

The deposition of the collagen fibers showed marked increase in subgroup I (Figure 2 B). However, the collagen deposition was reduced in subgroup II (Figure $2 \mathrm{C}$ ).

\section{Immunohistochemistry}

Examination of the sections of subgroup I revealed increased caspase-3, eNOS, iNOS, TNF- $\alpha$ immunoreactivity in the sarcoplasm of the cardiacmuscle fibers (Figures 3 B, 4 B, 5 B, 6 B) as well as increased eNOS immunostaining in the endothelium of the blood vessels (Figures $3 \mathrm{~A}, 4 \mathrm{~A}, \mathrm{~B}$, $5 \mathrm{~A}, 6 \mathrm{~A})$. However, these findings showed some improvement in subgroup II (Figures $3 \mathrm{C}, 4 \mathrm{C}$, 5 C, 6 C).

\section{Histomorphometric results}

The area percentage of the deposited collagen fibers, caspase-3, eNOS, iNOS and TNF- $\alpha$ immunostaining showed a significant increase in subgroup. A significant improvement in the area percentage of caspase-3, eNOS, iNOS, TNF- $\alpha$ immunoreactivity was detected in subgroup II. However, a non-significant decrease in the area percentage of the deposited collagen fibers was observed in subgroup II (Table I).

\section{Discussion}

It is challenging that up to $10 \%$ of patients treated with anthracyclines will develop cardiac complications that may manifest early or may emerge decades after the completion of therapy [8]. There has been mediocre success, at best, in preventing this toxic effect [1] and the prognosis of anthracycline-induced cardiomyopathy remains poor. There is a need to develop strategies and agents that can effectively prevent this toxicity. 

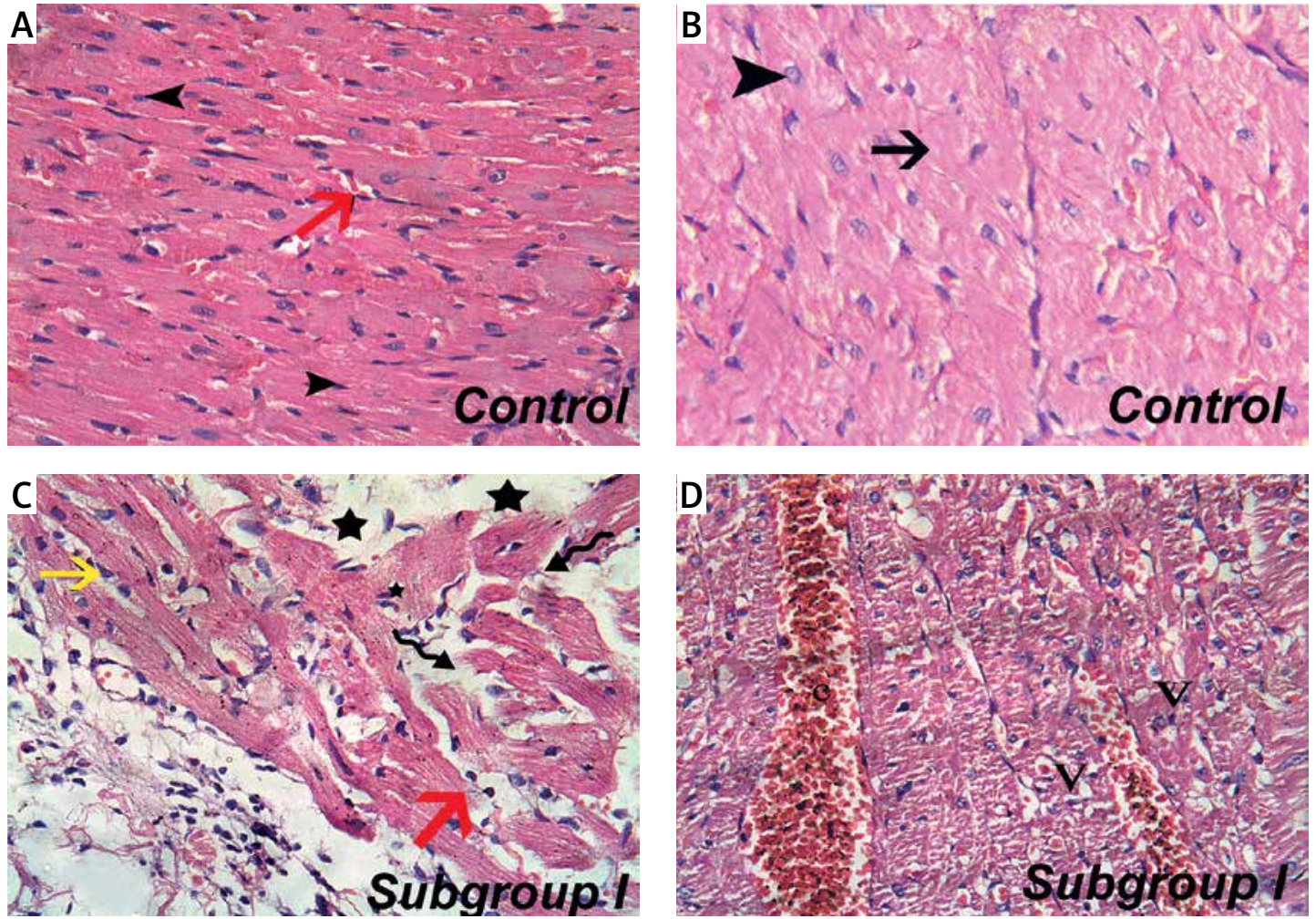

$\mathrm{E}$
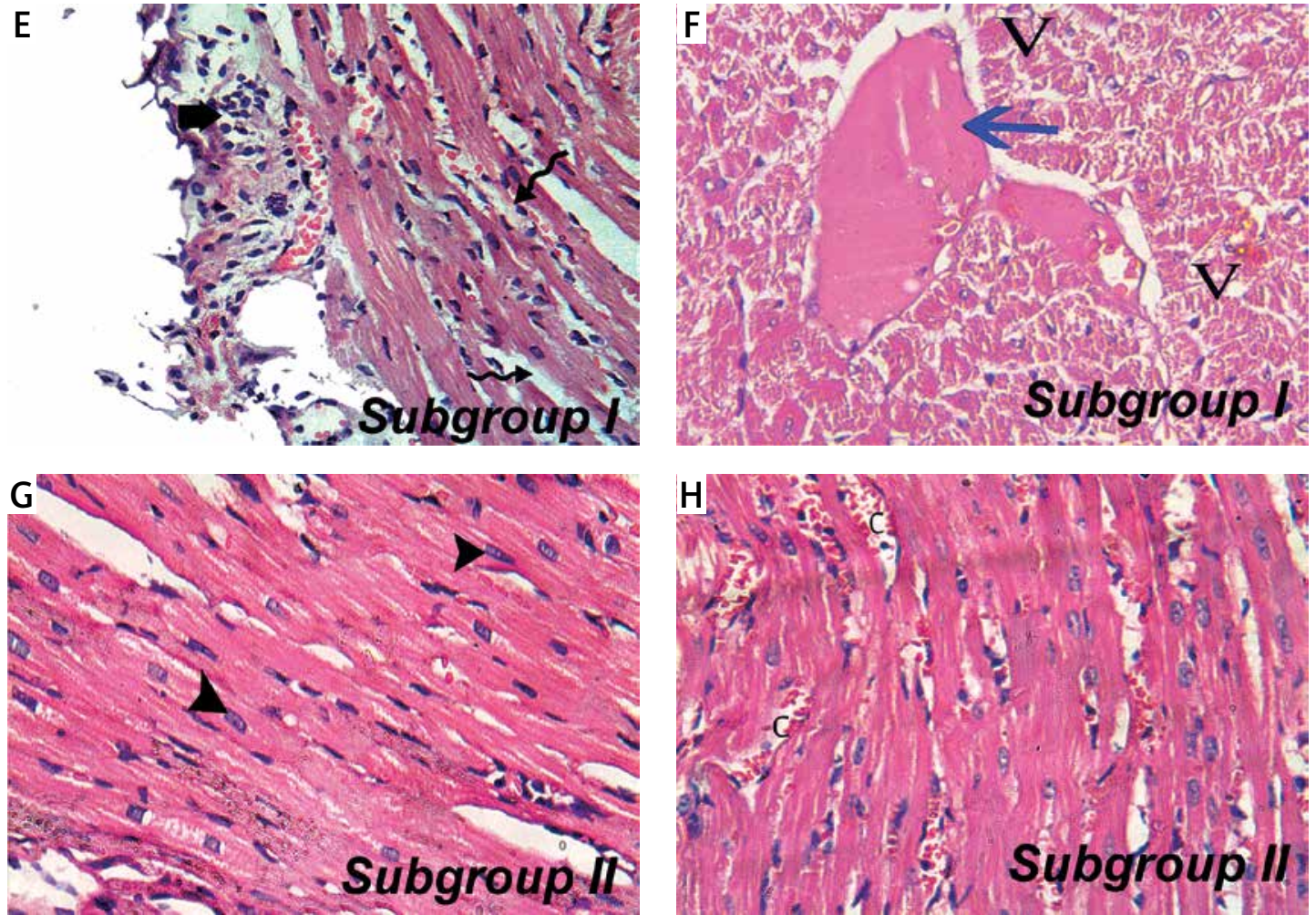

Figure 1. Photomicrograph of the left ventricle. A, B - control group showing the myocytes appeared cylindrical (arrow) with central oval nuclei (arrow heads) and blood capillaries (red arrow). C-F - subgroup I showing marked distortion (wavy arrows) and wide separation (stars) of cardiac myocytes. Some myocytes showed peripheral (yellow arrow) and pyknotic nuclei (red arrow). Inflammatory cell infiltrates (thick arrow), cytoplasmic vacuolation (V) of cardiac myocytes and massive congestion of the blood vessels (C) were evident. Lymph vessels were markedly dilated with acidophilic lymph (blue arrow). G, $\mathbf{H}$ - subgroup II showing regeneration of cardiac muscles fibers with few congested capillaries (C), (H+E, 400x) 

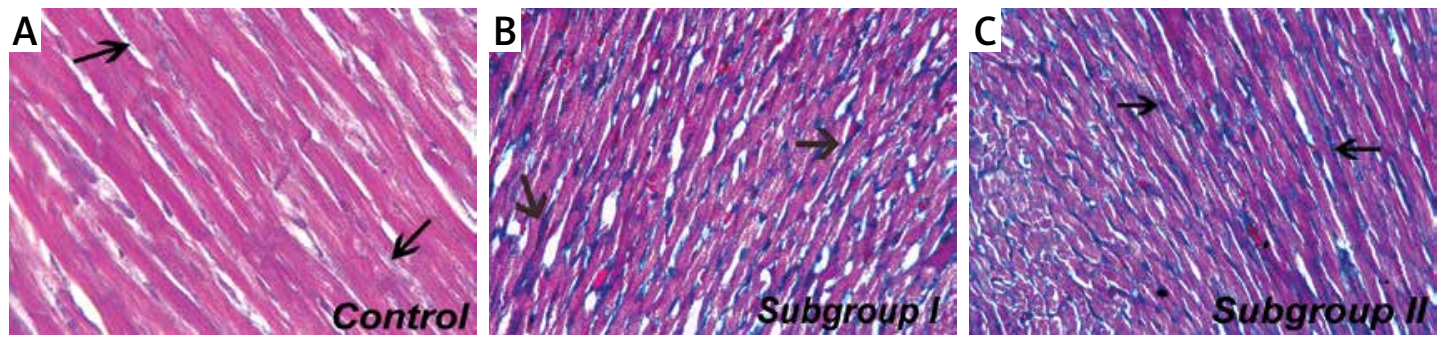

Figure 2. Photomicrograph of the left ventricle. A - Control group showing scanty collagen fibers between the cardiac myocytes. B - Subgroup I showing increased collagen deposition between the cardiac myocytes. C - subgroup II showing relative reduction in collagen deposition between cardiac myocytes. Arrows: collagen fibers (Masson's trichrome, 400x)
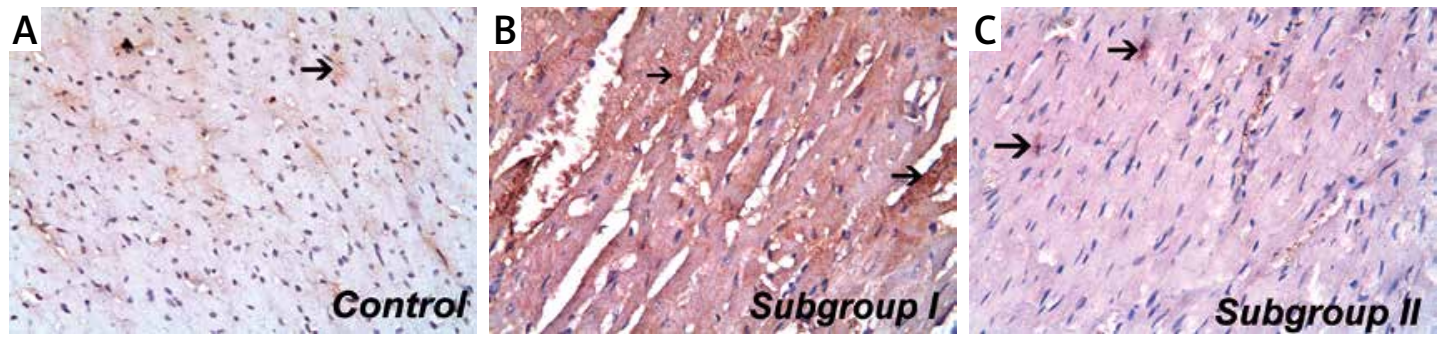

Figure 3. Photomicrograph of the left ventricle. A - Control group showing mild localized immunoreactivity in the sarcoplasm of cardiac myocytes. B - Subgroup I showing dense immunoreactivity in the sarcoplasm of cardiac myocytes. C - Subgroup II showing mild immunoreactivity in the sarcoplasm of cardiac myocytes. Arrows: immunoreactivity in sarcoplasm of cardiac myocytes (caspase-3, 400x)
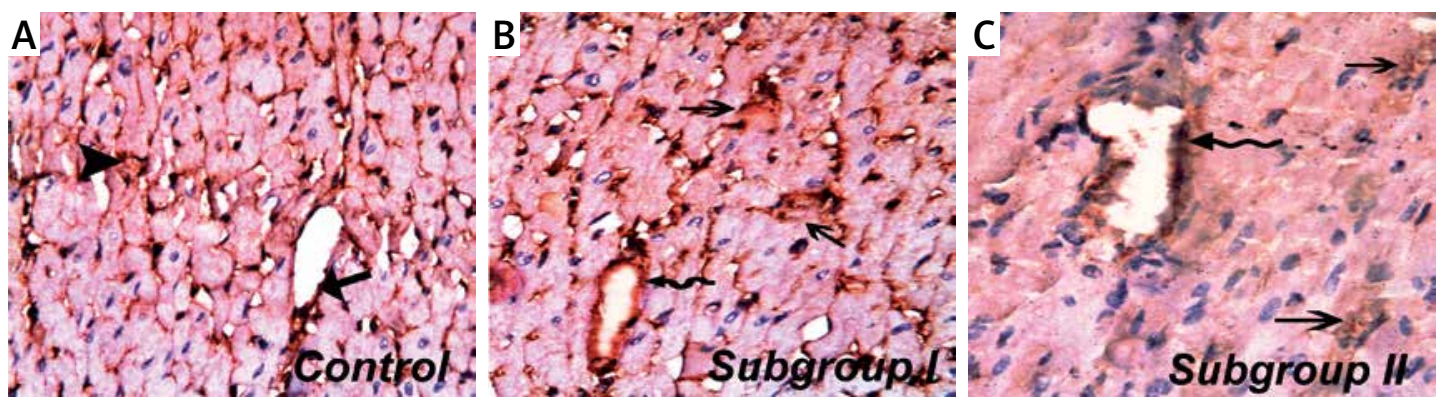

Figure 4. Photomicrograph of the left ventricle. A - Control group showing mild immunoreactivity in the sarcoplasm of cardiac myocytes (arrow head) as well as in the blood vessel endothelium (arrow). B - Subgroup I showing increased immunoreactivity in the sarcoplasm of cardiac myocytes (arrows) as well as in the blood vessel endothelium (wavy arrow). C - Subgroup II showing reduced immunoreactivity in the sarcoplasm of cardiac myocytes (arrows) as well as in the blood vessel endothelium (wavy arrow) (eNOS, 400x)
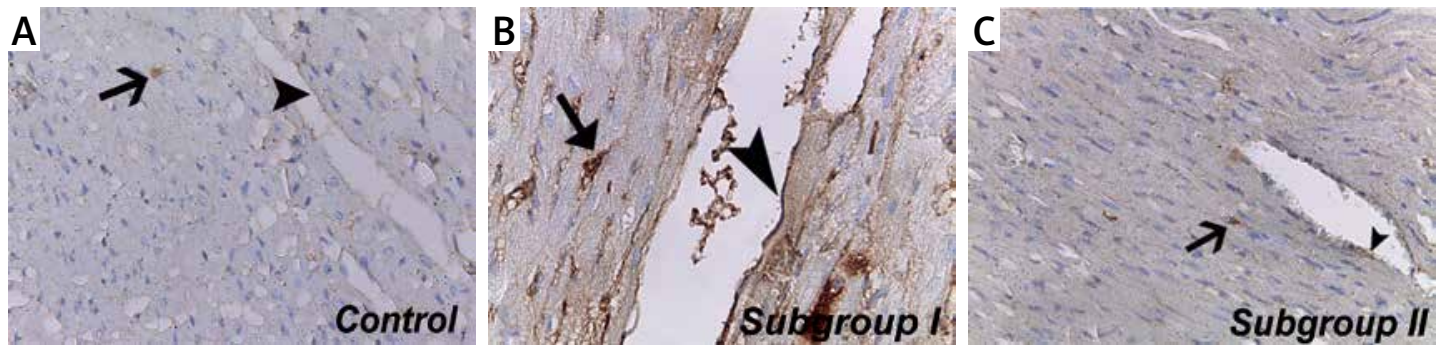

Figure 5. Photomicrograph of the left ventricle. A - Control group showing mild localized immunoreactivity in the sarcoplasm of cardiac myocytes as well as in the blood vessel endothelium. B - Subgroup I showing dense immunoreactivity in the sarcoplasm of cardiac myocytes as well as in the blood vessel endothelium. C - Subgroup II showing mild immunoreactivity in the sarcoplasm of cardiac myocytes as well as in the blood vessel endothelium. Arrows: immunoreactivity in sarcoplasm of cardiac myocytes, arrow head: immunoreactivity in the blood vessel endothelium (iNOS, 400x) 

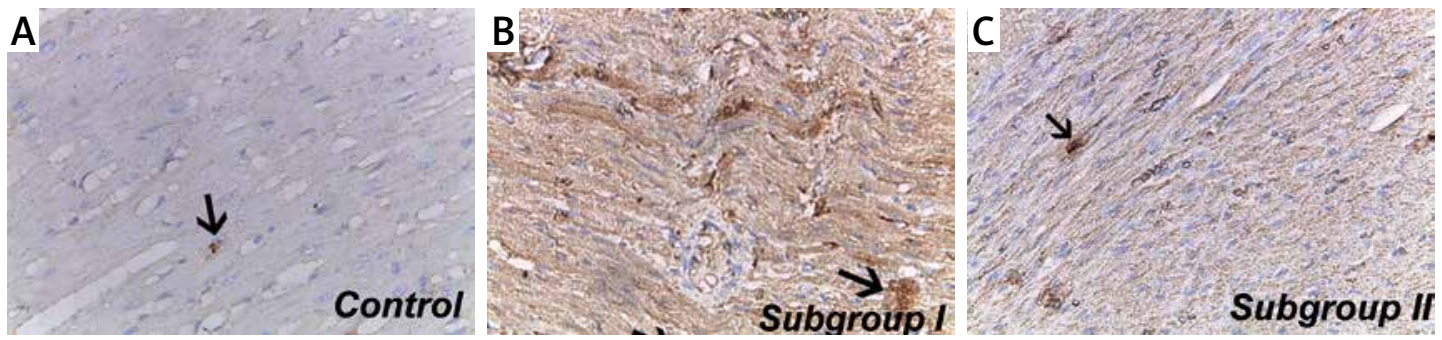

Figure 6. Photomicrograph of the left ventricle. A - Control group showing mild localized immunoreactivity in the sarcoplasm of cardiac myocytes. B - Subgroup I showing dense diffuse immunoreactivity in the sarcoplasm of cardiac myocytes. $\mathbf{C}$ - Subgroup II showing reduced immunoreactivity in the sarcoplasm of cardiac myocytes. Arrows: immunoreactivity in sarcoplasm of cardiac myocytes (TNF- $\alpha, 400 \times)$

We found that doxorubicin caused severe damage to the myocytes. This finding has been documented in several studies. Doxorubicin toxicity manifests as multifocal patchy areas, myofibrillar loss and vacuolated myocytes. Fibrotic foci generally predominate, whereas areas of acute damage are infrequent, although not absent. Foci of frank necrotic cardiomyocytes may be encountered [9]. These morphologic patterns are not specific to doxorubicin-induced cardiomyopathies, and they are seen across different species [9]. Nebivolol-treated rats were largely spared the histologic damage observed with doxorubicin treatment. To our knowledge, this is the first study to examine the effects of nebivolol pretreatment on the microscopic appearance of the heart. We believe the mechanisms we tested could explain the histological features observed with nebivolol pretreatment

We found an insignificant reduction in the extent of fibrous tissue deposition in rats treated with nebivolol. The antiproliferative effects of nebivolol are mediated by inhibition of the expression of inflammatory proteins, metalloproteinases and protease inhibitors. We think the insignificant results in our study could be due to the short time between induction of toxicity and histopathologic assessment. Collagen fiber deposition and fibrous tissue replacement are relatively late events in the process of maladaptive healing and can occur after a long time from an apparently uneventful exposure.

We also found that nebivolol pre-treatment significantly reduced the expression of caspase-3 immunoreactivity compared to doxorubicin treatment alone. Apoptotic cell death is a key component in doxorubicin-induced cardiotoxicity, but the exact triggers/mechanisms have not been fully established [10]. Caspase-3 plays a pivotal role in the apoptotic pathway. Doxorubicin, directly or indirectly, activates the caspase-3 pathway [8]. Our results can provide insight into a protective effect of nebivolol against doxorubicin-induced caspase activation and acceleration of apoptosis. Maejima et al. showed that administration of a NO donor suppressed caspase-3 activity and produced anti-apoptotic effects in cardiomyocytes treated with doxorubicin [11]. Nebivolol can function as a nitric oxide donor [13]. The anti-apoptotic effects of nebivolol were documented after myocardial infarction in rats by Mercanoglu et al., who found that nebivolol reduces myocardial apoptosis by preventing reactive oxygen species formation, therefore limiting expansion of the infarct zone and helping to maintain left ventricular function [14].

We found that doxorubicin significantly increased the expression of eNOS in the myocardium and that nebivolol pretreatment significantly attenuated this effect. The interaction between doxorubicin and NOS pathways is complex. Doxorubicin effectively transforms eNOS from a nitric oxide donor to a superoxide generator [8]. Doxorubicin increases eNOS transcription probably through doxorubicin-induced reactive oxygen species (ROS) formation [8]. Upregulated eNOS expression may play a key role in doxorubicin cardiotoxicity by mediating reactive oxygen species-induced apoptosis of endothelial cells [15]. Antisense eNOS mutation depresses doxorubicin-induced [16]. Genetic disruption of eNOS transcription protects against doxorubicin-induced oxidative stress and apoptosis and, subsequently, cardiac dysfunction and mortality, while overexpression of the same gene exaggerated the toxic effects of doxorubicin [17]. Human studies of endothelial dysfunction showed significant attenuation of endothelial-dependent and independent vasodilation after 30 min of doxorubicin administration, suggesting dysfunctional eNOS activity [18]. Our study provides, therefore, some proof that nebivolol attenuates doxorubicin toxicity via modulating the pathway ending in free radical generation. Nebivolol prevents eNOS uncoupling by reducing ROS, increasing NO bioavailability, and inhibiting up-regulation of the activity and expression of the vascular nicotinamide adenine dinucleotide phosphate oxidase [19].

We found the same changes with iNOS: a significant increase in expression with doxorubicin that was attenuated by nebivolol pretreatment. Mukho- 
Table I. Comparison of morphometric parameters among the different experimental groups

\begin{tabular}{|c|c|c|c|}
\hline Variable & Reference group & Experimental group & $P$-value \\
\hline \multirow{6}{*}{$\begin{array}{l}\text { Mean area percentage } \\
\text { of fibrosis }\end{array}$} & \multirow[t]{2}{*}{ Control $(7.66 \pm 0.84)$} & Doxorubicin-treated group & $0.04^{*}$ \\
\hline & & Doxorubicin + nebivolol-treated group & 1 \\
\hline & \multirow{2}{*}{$\begin{array}{l}\text { Doxorubicin-treated group } \\
\qquad(18.87 \pm 8.46)\end{array}$} & Control & $0.04^{\star}$ \\
\hline & & Doxorubicin + nebivolol-treated group & 0.07 \\
\hline & \multirow{2}{*}{$\begin{array}{l}\text { Doxorubicin + nebivolol- } \\
\text { treated group }(12.81 \pm 5.82)\end{array}$} & Control & 1 \\
\hline & & Doxorubicin-treated group & 0.07 \\
\hline \multirow{6}{*}{$\begin{array}{l}\text { Mean area percentage } \\
\text { of caspase- } 3 \\
\text { immunoreactivity }\end{array}$} & \multirow[t]{2}{*}{ Control $(4.42 \pm 0.99)$} & Doxorubicin-treated group & $0.001^{*}$ \\
\hline & & Doxorubicin + nebivolol-treated group & 0.03 \\
\hline & \multirow{2}{*}{$\begin{array}{l}\text { Doxorubicin-treated group } \\
\qquad(27.44 \pm 5.94)\end{array}$} & Control & $0.001^{*}$ \\
\hline & & Doxorubicin + nebivolol-treated group & 0.03 \\
\hline & \multirow{2}{*}{$\begin{array}{l}\text { Doxorubicin + nebivolol- } \\
\text { treated group }(15.94 \pm 2.81)\end{array}$} & Control & 0.03 \\
\hline & & Doxorubicin-treated group & 0.03 \\
\hline \multirow{6}{*}{$\begin{array}{l}\text { Mean area } \\
\text { percentage of iNOS } \\
\text { immunoreactivity }\end{array}$} & \multirow[t]{2}{*}{ Control $(0.54 \pm 1.06)$} & Doxorubicin-treated group & $<0.001^{*}$ \\
\hline & & Doxorubicin + nebivolol-treated group & $<0.001^{*}$ \\
\hline & \multirow{2}{*}{$\begin{array}{l}\text { Doxorubicin-treated group } \\
\qquad(2.46 \pm 10.27)\end{array}$} & Control & $<0.001^{*}$ \\
\hline & & Doxorubicin + nebivolol-treated group & $<0.001^{*}$ \\
\hline & \multirow{2}{*}{$\begin{array}{l}\text { Doxorubicin + nebivolol- } \\
\text { treated group }(0.51 \pm 1.25)\end{array}$} & Control & $<0.001^{*}$ \\
\hline & & Doxorubicin-treated group & $<0.001^{*}$ \\
\hline \multirow{6}{*}{$\begin{array}{l}\text { Mean area } \\
\text { percentage of TNF- } \alpha \\
\text { immunoreactivity }\end{array}$} & \multirow[t]{2}{*}{ Control $(0.42 \pm 0.85)$} & Doxorubicin-treated group & $<0.001^{*}$ \\
\hline & & Doxorubicin + nebivolol-treated group & $<0.001^{*}$ \\
\hline & \multirow{2}{*}{$\begin{array}{l}\text { Doxorubicin-treated group } \\
\qquad(5.83 \pm 43.4)\end{array}$} & Control & $<0.001^{*}$ \\
\hline & & Doxorubicin + nebivolol-treated group & $0.003^{*}$ \\
\hline & \multirow{2}{*}{$\begin{array}{l}\text { Doxorubicin + nebivolol- } \\
\text { treated group }(0.91 \pm 1.53)\end{array}$} & Control & $<0.001^{*}$ \\
\hline & & Doxorubicin-treated group & $0.003^{*}$ \\
\hline \multirow{6}{*}{$\begin{array}{l}\text { Mean area } \\
\text { percentage of eNOS } \\
\text { immunoreactivity }\end{array}$} & \multirow[t]{2}{*}{ Control $(4.1 \pm 0.3)$} & Doxorubicin-treated group & $0.04^{*}$ \\
\hline & & Doxorubicin + nebivolol-treated group & $0.001^{*}$ \\
\hline & \multirow{2}{*}{$\begin{array}{l}\text { Doxorubicin-treated group } \\
\qquad(15.2 \pm 3.5)\end{array}$} & Control & $0.04^{*}$ \\
\hline & & Doxorubicin + nebivolol-treated group & $<0.001^{*}$ \\
\hline & \multirow{2}{*}{$\begin{array}{l}\text { Doxorubicin + nebivolol- } \\
\text { treated group }(8.6 \pm 1.4)\end{array}$} & Control & $<0.001^{*}$ \\
\hline & & Doxorubicin-treated group & $<0.001^{*}$ \\
\hline
\end{tabular}

*Statistically significant.

padhyay et al. also found a significant increase in iNOS expression with doxorubicin that was attenuated by ROS scavenger. They proposed that iNOS is the predominant source of doxorubicin-induced increased NO formation to generate reactive oxygen species in the mitochondria and cytosol with consequent mitochondrial damage [10].

TNF is a pleiotropic cytokine that binds to receptors TNF-R1 and TNF-R2 and, depending on cell type, triggers different signaling pathways, including cell death and inflammation. Doxorubicin is a potent inducer of apoptosis in cardiomyocytes that is accompanied by relevant changes in TNF- $\alpha$ receptor levels. Moreover, treatment with exogenous TNF- $\alpha$ strongly potentiates the apoptotic effect of doxorubicin in cardiomyocytes [20]. TNF- $\alpha$ enhances the effect of doxorubicin on cancer cells via efficient induction of caspase-8 cleav- 
age promoting cell death [21]. Doxorubicin-killed cells could also be a source of further TNF- $\alpha$ production, which would amplify the inflammatory response [22]. Our results contradict Górska et al., who reported that nebivolol did not modify serum concentrations of TNF- $\alpha$ in hypertensive rats [23]. In addition, Chiosi et al. showed no change in serum concentration of cytokines including TNF in heart failure patients treated with nebivolol [20]. The differences could have resulted from sampling the plasma versus sampling the myocardium.

There has been only one available animal experiment that investigated the protective effect of nebivolol from doxorubicin cardiotoxicity. De Nigris et al. found in a model of isolated perfused rat heart that nebivolol compared to carvedilol, a $\beta$-blocker with anti-oxidant properties, exerted the most significant cardioprotective effects in terms of left ventricular diastolic pressure and rate of pressure rise [24].

Our study is of clinical interest as $\beta$-blockers appeared to provide more protection than ACE inhibitors and statins in human clinical studies of anthracycline cardiotoxicity. In lymphoma-treated doxorubicin patients, heart failure was less frequent under concomitant treatment with either ACE inhibitor or metoprolol than no treatment, especially in the metoprolol group [25]. Carvedilol preserved the diastolic functions and left ventricular diameters as compared to placebo in 25 patients treated with doxorubicin [26]. We found only one clinical trial that tested nebivolol as a prophylactic agent with doxorubicin. Kaya et al. allocated 45 consecutive patients with breast cancer and planned chemotherapy to receive nebivolol or placebo. The nebivolol group had lower systolic and diastolic dimensions, lower levels of natriuretic peptide and better ejection fraction, which all prove a protective effect for nebivolol [27]. To our knowledge, this is the first study to observe, with histopathology and immunohistochemistry, multiple postulated mechanisms of doxorubicin and nebivolol myocardial effects [28]. The used dosages of doxorubicin and nebivolol in rats can be compared to the usual dosages in humans through prospective randomized human clinical trial studies.

In conclusion, nebivolol reduced apoptosis, prevented maladaptive eNOS/iNOS activation and TNF- $\alpha$ expression and helped to reduce extracellular matrix deposition. Nebivolol is a drug that is relatively cheap, has a very favorable side effect profile, and is administered as a single daily dose. This makes it a good choice as a prophylactic agent. We recommend a statistically powered prospective randomized human clinical trial that compares nebivolol in different doses, alone or combined with other agents against placebo to prevent cardiotoxicity in patients who will receive doxorubicin.

\section{Conflict of interest}

The authors declare no conflict of interest.

\section{References}

1. Kalam K, Marwick TH. Role of cardioprotective therapy for prevention of cardiotoxicity with chemotherapy: a systematic review and meta-analysis. Eur J Cancer 2013; 49: 2900-9.

2. Chatterjee K, Zhang J, Honbo N, Karliner JS. Doxorubicin cardiomyopathy. Cardiology 2010; 115: 155-62.

3. Aryal B, Jeong J, Rao VA. Doxorubicin-induced carbonylation and degradation of cardiac myosin binding protein C promote cardiotoxicity. Proc Natl Acad Sci USA 2014; 111: 2011-6.

4. Ichikawa $Y$, Ghanefar M, Bayeva M, et al. Cardiotoxicity of doxorubicin is mediated through mitochondrial iron accumulation. J Clin Investig 2014; 124: 617-30.

5. Howlett JG. Nebivolol: vasodilator properties and evidence for relevance in treatment of cardiovascular disease. Can J Cardiol 2014; 30 (5 Suppl): S29-37.

6. Imbaby S, Ewais M, Essawy S, Farag N. Cardioprotective effects of curcumin and nebivolol against doxorubicin-induced cardiac toxicity in rats. Hum Exp Toxicol 2014; 33: 800-13.

7. Kaya MG, Ozkan M, Gunebakmaz O, et al. Protective effects of nebivolol against anthracycline-induced cardiomyopathy: a randomized control study. Int J Cardiol 2013; 167: 2306-10.

8. Octavia Y, Tocchetti CG, Gabrielson KL, Janssens S, Crijns HJ, Moens AL. Doxorubicin-induced cardiomyopathy: from molecular mechanisms to therapeutic strategies. J Mol Cell Cardiol 2012; 52: 1213-25.

9. Takemura G, Fujiwara H. Doxorubicin-induced cardiomyopathy from the cardiotoxic mechanisms to management. Prog Cardiovasc Dis 2007; 49: 330-52.

10. Mukhopadhyay P, Rajesh M, Bátkai S, et al. Role of superoxide, nitric oxide, and peroxynitrite in doxorubicin-induced cell death in vivo and in vitro. Am J Physiol Heart Circ Physiol 2009; 296: H1466-83.

11. Maejima Y, Adachi S, Morikawa K, Ito H, Isobe M. Nitric oxide inhibits myocardial apoptosis by preventing caspase-3 activity via S-nitrosylation. J Mol Cell Cardiol 2005; 38: 163-74.

12. Petrie A, Sabin C. Medical Statistics at a Glance. $2^{\text {nd }}$ ed. Sugden M, Moore K (eds.). Blackwell Publishing LTD., USA 2005; 55.

13. Maffei A, Di Pardo A, Carangi R, et al. Nebivolol induces nitric oxide release in the heart through inducible nitric oxide synthase activation. Hypertension 2007; 50: 652-6.

14. Mercanoglu G, Safran N, Gungor M, et al. The effects of nebivolol on apoptosis in a rat infarct model. Circ J 2008; 72: 660-70.

15. Vasquez-Vivar J, Martasek P, Hogg N, Masters BS, Pritchard KA Jr, Kalyanaraman B. Endothelial nitric oxide synthase-dependent superoxide generation from adriamycin. Biochemistry 1997; 36: 11293-7.

16. Kalivendi SV, Kotamraju S, Zhao H, Joseph J, Kalyanaraman B. Doxorubicin-induced apoptosis is associated with increased transcription of endothelial nitric-oxide 
synthase: effect of antiapoptotic antioxidants and calcium. J Biol Chem 2001; 276: 47266-76.

17. Neilan TG, Blake SL, Ichinose F, et al. Disruption of nitric oxide synthase 3 protects against the cardiac injury, dysfunction, and mortality induced by doxorubicin. Circulation 2007; 116: 506-14.

18. Duquaine D, Hirsch GA, Chakrabarti A, et al. Rapid-onset endothelial dysfunction with adriamycin: evidence for a dysfunctional nitric oxide synthase. Vasc Med 2003; 8: 101-7.

19. Oelze M, Daiber A, Brandes RP, et al. Nebivolol inhibits superoxide formation by NADPH oxidase and endothelial dysfunction in angiotensin II-treated rats. Hypertension 2006; 48: 677-84.

20. Chiosi E, Spina A, Sorrentino A, et al. Change in TNF-alpha receptor expression is a relevant event in doxorubicin-induced H9c2 cardiomyocyte cell death. J Interferon Cytokine Res 2007; 27: 589-97.

21. Cao W, Ma SL, Tang J, Shi J, Lu Y. A combined treatment TNF-alpha/doxorubicin alleviates the resistance of MCF-7/Adr cells to cytotoxic treatment. Biochim Biophys Acta 2006; 1763: 182-7.

22. Kaczmarek A, Krysko O, Heyndrickx L, et al. TNF/TNF-R1 pathway is involved in doxorubicin-induced acute sterile inflammation. Cell Death Dis 2013; 4: e961.

23. Górska D, Dudarewicz M, Czarnecka E, Andrzejczak D. Does nebivolol influence serum concentrations of proinflammatory cytokines in hypertensive (SHR) and normotensive (WKY) rats? Pharmacol Rep 2010; 62: 86-94.

24. de Nigris F, Rienzo M, Schiano C, Fiorito C, Casamassimi A Napoli C. Prominent cardioprotective effects of third generation beta blocker nebivolol against anthracycline-induced cardiotoxicity using the model of isolated perfused rat heart. Eur J Cancer 2008; 44: 334-40.

25. Georgakopoulos P, Roussou P, Matsakas E, et al. Cardioprotective effect of metoprolol and enalapril in doxorubicin-treated lymphoma patients: a prospective, parallel-group, randomized, controlled study with 36-month follow-up. Am J Hematol 2010; 85: 894-6.

26. Kalay N, Basar E, Ozdogru I, et al. Protective effects of carvedilol against anthracycline-induced cardiomyopathy. J Am Coll Cardiol 2006; 48: 2258-62.

27. Kaya MG, Ozkan M, Gunebakmaz O, et al. Protective effects of nebivolol against anthracycline-induced cardiomyopathy: a randomized control study. Int J Cardiol 2013; 167: 2306-10.

28. Munzel T, Gori T. Nebivolol: the somewhat-different beta-adrenergic receptor blocker. J Am Coll Cardiol 2009; 54: 1491-9. 